\title{
openheart Association of ambulatory heart rate and atherosclerosis risk factors with blood pressure in young non- hypertensive adults
}

\author{
Cynthia Cheng, ${ }^{1}$ Constantine Daskalakis ${ }^{2}$
}

To cite: Cheng C, Daskalakis C. Association of ambulatory heart rate and atherosclerosis risk factors with blood pressure in young non-hypertensive adults. Open Heart 2016;3:e000332. doi:10.1136/openhrt-2015000332

Received 28 August 2015 Revised 24 October 2015 Accepted 5 December 2015

\section{(1) CrossMark}

${ }^{1}$ Department of Family and Community Medicine, Thomas Jefferson University, Philadelphia, Pennsylvania, USA

${ }^{2}$ Division of Biostatistics, Department of Pharmacology and Experimental

Therapeutics, Thomas Jefferson University, Philadelphia, Pennsylvania, USA

Correspondence to Dr Cynthia Cheng; Cynthia.Cheng@jefferson.edu

\section{ABSTRACT}

Objective: The study objective was to assess the association between $24 \mathrm{~h}$ ambulatory heart rate (HR), atherosclerosis risk factors and blood pressure (BP) in young non-hypertensive patients.

Methods: We recruited 186 participants aged 1845 years from a large urban academic Family Medicine outpatient practice, serving 40000 individuals for this observational study. The main analyses were based on multiple linear regression, with mean $24 \mathrm{~h} \mathrm{BP}$ (systolic $\mathrm{BP}(\mathrm{SBP})$ or diastolic BP (DBP)) as the outcomes, mean $24 \mathrm{~h} \mathrm{HR}$ as the main predictor of interest, and controlling for age, gender, race, insulin sensitivity/ resistance and endothelial function measured by strain gauge venous occlusion plethysmography.

Results: HR was independently associated with mean $24 \mathrm{~h} \mathrm{SBP}$ and DBP (SBP and DBP: $p=0.042$ and 0.001 , respectively). In our analyses, associations were markedly stronger for ambulatory compared with office BP measurements. Endothelial dysfunction was associated with higher SBP $(p=0.013)$; plasminogen activator inhibitor-1 was significantly associated with both SBP and DBP ( $p=0.041$ and 0.015 , respectively), while insulin resistance was not associated with either $\mathrm{SBP}$ or DBP. Insulin resistance and $\mathrm{C}$ reactive protein were significant predictors of HR $(p=0.013$ and 0.007 , respectively).

Conclusions: These findings suggest that HR may be a potential marker of elevated cardiovascular risk in young asymptomatic individuals, prior to the development of clinical hypertension or cardiovascular disease.

\section{INTRODUCTION}

Heart rate (HR) is a key determinant of coronary blood flow, an established marker of sympathetic nervous system (SNS) activity, and an independent predictor of cardiovascular morbidity and mortality in various adult patient populations with and without cardiovascular disease. $^{1}$ Increased SNS activity increases blood pressure (BP) and contributes to the development and maintenance of hypertension. ${ }^{2}$ Multiple studies have demonstrated a

\section{KEY QUESTIONS}

What is already known about this subject?

- Resting heart rate (HR) is an independent predictor of cardiovascular and all-cause mortality in men and women with and without diagnosed cardiovascular disease.

What does this study add?

- Most previous studies of HR have utilised resting HR. Three previous studies which analysed mean $24 \mathrm{~h} \mathrm{HR}$, as in our study, recruited individuals much older than those in our study. Our findings suggest that HR may be a potential marker of elevated cardiovascular risk in asymptomatic individuals, prior to the development of clinical hypertension or cardiovascular disease.

How might this impact on clinical practice?

- Although epidemiological studies have consistently reported that resting HR is an independent predictor of cardiovascular and all-cause mortality, the importance of resting HR as a prognostic factor and potential therapeutic target is not yet generally accepted. Clinical strategies incorporating HR in risk stratification may have possible future utility in identifying asymptomatic individuals to target with individualised preventive therapy.

correlation between increased SNS activity and higher BP elevation in patients with essential hypertension; this association is not present in secondary hypertension. ${ }^{3}$ Furthermore, elevated $H R$ is present in a significant proportion of adult borderline hypertensives, beginning in childhood. ${ }^{4}$ These observations suggest that sympathetic activation is a specific feature of essential hypertension and that heightened SNS activity may play a pathogenetic role in hypertension pathophysiology and prognosis. ${ }^{5}$

It has been hypothesised that increased SNS activity may contribute to the pathogenesis of elevated BP via direct vasoconstrictive 
effects on blood vessels, via stimulation of release of adipokines and/or via SNS-mediated insulin resistance. ${ }^{6}$ Up to $70 \%$ of newly diagnosed hypertensive cases are attributable to obesity ${ }^{7}$; increased kidney and muscle sympathetic nerve activity (SNA) are found in obesity. ${ }^{8}$ Muscle SNA levels decline with weight loss and increase with weight gain. ${ }^{9}$ Compared with subcutaneous or lower body fat, visceral abdominal fat, which is the primary source of $\mathrm{C}$ reactive protein (CRP) and other proinflammatory adipokines, is more closely associated with increased resting HR and higher SNA in humans. ${ }^{10}$

Elevated HR has deleterious cardiovascular consequences in addition to elevation of BP. High resting HR may potentially accelerate atherosclerosis via its negative effects on the endothelium. ${ }^{11}$ There is a close relationship between shear stress and $\mathrm{HR}^{1}$; accelerated HR may promote endothelial shear stress.

To further investigate the potential role of HR in the early pathophysiology of atherosclerosis, we analysed data from a cohort of non-hypertensive young adults. The primary study objective was to evaluate the direct link between ambulatory $24 \mathrm{~h} \mathrm{HR}$ and ambulatory $24 \mathrm{~h}$ BP (figure 1, pathway [1]), accounting for other potential risk factors that may also independently impact BP, including endothelial dysfunction, insulin resistance, inflammatory markers and measures of adiposity (figure 1, pathways [2]-[5]). The secondary study objective was to assess potential determinants of HR (figure 1, pathways [6]-[8]).

\section{RESEARCH METHODS AND PROCEDURES Participants/study design}

Data were collected using an observational crosssectional study design from a cohort of volunteers enrolled in an ongoing investigation of the role of adipokines and insulin resistance in the pathogenesis of hypertension. Participants were recruited from a large urban academic Family Medicine outpatient practice, serving 40000 individuals in Philadelphia, Pennsylvania, USA. Inclusion criteria were age between 18 and 45 years and absence of hypertension: office systolic BP (SBP) $<140 \mathrm{~mm} \mathrm{Hg}$ and diastolic BP (DBP) $<90 \mathrm{~mm} \mathrm{Hg}$. Exclusion criteria were diabetes, pregnancy, hypertension, coronary or cerebrovascular disease, collagen vascular disease, organ failure (heart, kidney, liver), and statin medication use. The study was approved by the Thomas Jefferson University Institutional Review Board, and written informed consent was obtained from all participants.

\section{Measurements and data collection}

Information was collected on sociodemographic characteristics and current health behaviours. Height and weight were measured and body mass index (BMI) was calculated as weight divided by height squared $\left(\mathrm{kg} / \mathrm{m}^{2}\right)$.

\section{$\mathrm{BP}$ and $\mathrm{HR}$}

Resting SBP and DBP and HR were measured at the office with a Dinamap cuff. In addition, the participants were also provided with an oscillometric device (Spacelabs 90217A, Spacelabs) for $24 \mathrm{~h}$ ambulatory BP monitoring $(\mathrm{ABPM})$, with readings set at $20 \mathrm{~min}$ intervals while awake, and $60 \mathrm{~min}$ intervals while asleep. Mean $24 \mathrm{~h} \mathrm{SBP,} \mathrm{DBP}$ and HR were calculated, as well as the means of these measures during the awake and asleep periods. SBP, DBP and HR variability were also

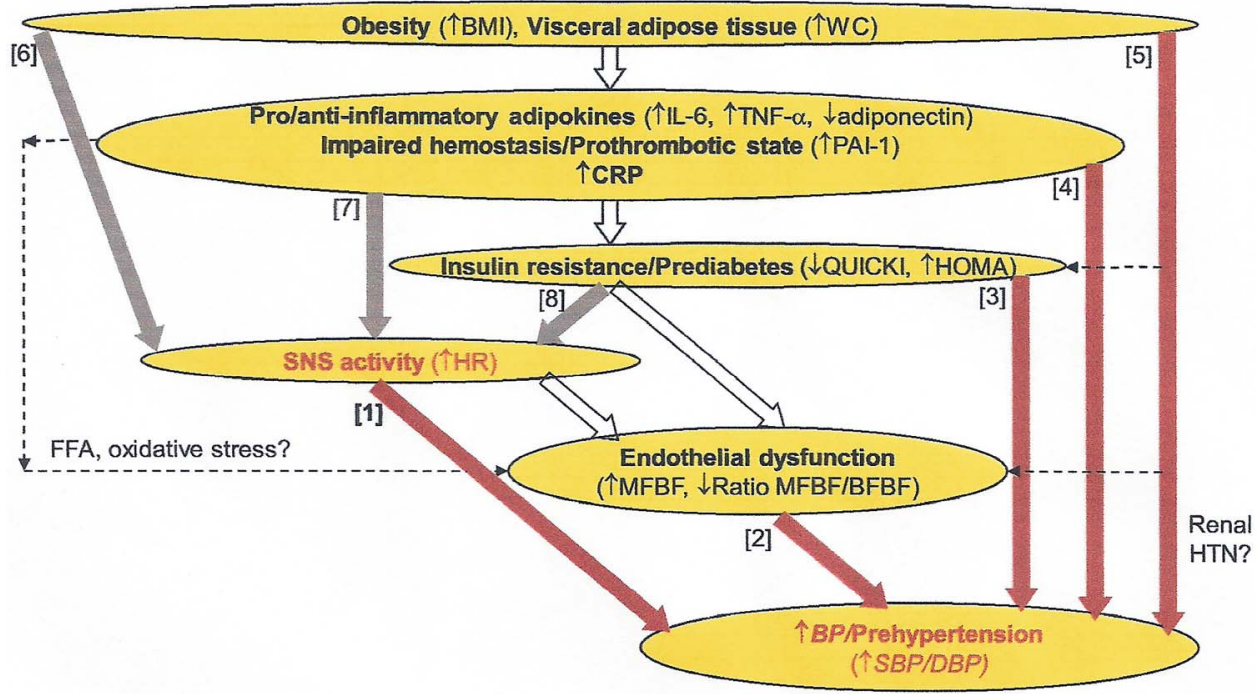

Figure 1 Diagram of potential causal pathways linking atherosclerotic risk factors. BFBF, baseline forearm blood flow; BMI, body mass index; DBP, diastolic blood pressure; FFA, free fatty acids; HOMA, homoeostatic model assessment; HR, heart rate; HTN, hypertension; IL-6, interleukin-6; MFBF, maximum forearm blood flow; PAI-1, plasminogen activator inhibitor-1; SBP, systolic blood pressure; SNS, sympathetic nervous system; TNF, tomour necrosis factor; QUICKI, quantitative insulin sensitivity check index; WC, waist circumference. 
computed, as the within-participant SD of the ambulatory measurements.

\section{Endothelial function}

Minimal forearm vascular resistance (MFVR) at maximal vasodilation is considered an integrated measure of vascular resistance in the forearm. ${ }^{12}$ We assessed forearm vascular resistance and forearm blood flow noninvasively using plethysmography according to the method of Sivertsson. ${ }^{13}$ First, we measured baseline forearm blood flow (BFBF) at rest and maximum forearm blood flow (MFBF) during postocclusive reactive hyperaemia. We then calculated both baseline forearm vascular resistance (BFVR=baseline mean arterial pressure (MAP) divided by BFBF) and minimum forearm vascular resistance $(\mathrm{MFVR}=$ postocclusive MAP divided by MFBF). The main measures used in the analyses were the per cent increase in FBF and the per cent decrease in FVR (computed as (MFBF-BFBF)/ $\mathrm{BFBF} \times 100$ and $(\mathrm{BFVR}-\mathrm{MFVR}) / \mathrm{BFVR} \times 100$, respectively). Several groups have assessed the reproducibility of venous occlusion plethysmography. Roberts et al (1986) demonstrated good within-participant reproducibility for unilateral blood flow measurement, used in this study, with a coefficient of variation of $\sim 10 \%$.

\section{Insulin resistance}

Fasting plasma samples were obtained following an overnight fast and analysed using a Glucostat analyser (YSI, Model 27). Insulin was assayed using a solid phase radioimmunoassay, 'Coat-A-Count' (Diagnostic Products Corporation). We computed two common indices of insulin sensitivity. The homoeostatic model assessment (HOMA) index was computed as the product of fasting glucose $(\mathrm{mM} / \mathrm{L})$ and fasting insulin $(\mathrm{uU} / \mathrm{mL})$, divided by 22.5. The quantitative insulin sensitivity check index (QUICKI) was computed as the reciprocal of the sum of the $\log$ fasting glucose $(\mathrm{mg} / \mathrm{dL})$ and the $\log$ fasting insulin $(\mathrm{uU} / \mathrm{mL})$. We used QUICKI in the main analyses, as it tends to have a more symmetric (normal) distribution than the HOMA and does not require logarithmic transformation. ${ }^{14}$

\section{Inflammatory markers}

We measured five inflammatory markers: plasminogen activator inhibitor-1 (PAI-1), interleukin 6 (IL-6), tumour necrosis factor $\alpha(\mathrm{TNF} \alpha)$, adiponectin and highsensitive CRP. Assays were performed using commercially available ELISA kits, which have coefficients of variation $4-6 \%$.

\section{Statistical analyses}

The main study objective was to assess the association of HR and HR variability with BP (figure 1, pathway [1]), controlling for other risk factors, mainly endothelial function and insulin resistance (figure 1, pathways [2] and [3]). The main analyses were based on multiple linear regression, with mean $24 \mathrm{~h} \mathrm{BP}$ (SBP or DBP) as the outcomes, mean $24 \mathrm{~h} \mathrm{HR}$ or HR variability as the main predictor of interest, and controlling for age, gender, race, endothelial function and insulin sensitivity/resistance. Although endothelial dysfunction and insulin resistance may develop as a consequence of hypertension, they are also possible contributors to the pathogenesis of hypertension. Therefore, we controlled for them in order to isolate the independent association between HR and BP. Additional analyses also controlled for inflammatory markers and measures of adiposity (figure 1, pathways [4] and [5]). We also carried out similar analyses with office measurements of $\mathrm{BP}$ and $\mathrm{HR}$, instead of the $24 \mathrm{~h}$ ambulatory averages.

The secondary study objective was to evaluate the extent to which HR is impacted by other atherosclerotic risk factors, specifically measures of adiposity, inflammation and insulin resistance (figure 1, pathways [6] through [8]). The main analyses were again based on multiple linear regression, with mean $24 \mathrm{~h} \mathrm{HR}$ as the outcome, and measures of adiposity, inflammatory markers and insulin sensitivity/resistance as the predictors of interest.

Because of their skewed distributions, IL-6, adiponectin, CRP and FVR per cent decrease were logtransformed in all analyses. Statistical analyses were conducted in SAS V.9.4 (SAS Institute; Cary, North Carolina, USA). The study was powered at $80 \%$ or higher for detecting outcomes as noted for these analyses. Missing data were not imputed.

\section{RESULTS}

The analyses were based on data from 186 participants. Ambulatory BP and HR measurements were based on an average of 42 readings per participant for the awake period and 8 readings per participant for the asleep period ( $\mathrm{SD}=9$ and 2 , respectively). Table 1 summarises the characteristics of the study participants. No participant was hypertensive, while 44 (24\%) were prehypertensive by either their office BP (ie, office SBP $120-139 \mathrm{~mm} \mathrm{Hg}$ or DBP $80-89 \mathrm{~mm} \mathrm{Hg}$ ) or their $24 \mathrm{~h}$ BP (ie, $24 \mathrm{~h}$ SBP $130-134 \mathrm{~mm} \mathrm{Hg}$ or $24 \mathrm{~h} \mathrm{DBP}$ $80-84 \mathrm{~mm} \mathrm{Hg})$. These included $8(4 \%)$ classified as prehypertensive by both office and $24 \mathrm{~h}$ BP, $18(10 \%)$ with white coat prehypertension (ie, office but not $24 \mathrm{~h}$ ) and $18(10 \%)$ with masked prehypertension (ie, $24 \mathrm{~h}$ but not office). Finally, 66 participants $(35 \%)$ were 'SBP non-dippers', that is, their ambulatory mean SBP had dropped less than $10 \%$ from their awake to their sleep period.

Table 2 presents the correlations of mean $24 \mathrm{~h}$ BP and the various risk factors, including mean $24 \mathrm{~h} \mathrm{HR}$ and HR variability. HR was a modest predictor of DBP but not SBP. By contrast, endothelial dysfunction, measured as the FBF per cent increase or the FVR per cent decrease, was significantly associated with SBP but not DBP. Insulin sensitivity (QUICKI) was significantly and inversely related with both SBP and DBP. All these 
Table 1 Study participant characteristics $(\mathrm{N}=186)$

\begin{tabular}{|c|c|}
\hline Age (years), mean (SD) & $27(6)$ \\
\hline \multicolumn{2}{|l|}{ Gender, n (\%) } \\
\hline Female & $115(62)$ \\
\hline Male & $71(38)$ \\
\hline \multicolumn{2}{|l|}{ Race, n (\%) } \\
\hline Caucasian & $98(53)$ \\
\hline Black/African-American & $32(17)$ \\
\hline Hispanic/Latino & $10(5)$ \\
\hline Asian & $46(25)$ \\
\hline BMI, $\mathrm{kg} / \mathrm{m}^{2},{ }^{*}$ mean (SD) & $25(5)$ \\
\hline Waist circumference $(\mathrm{cm}), \uparrow$ mean $(\mathrm{SD})$ & $83(12)$ \\
\hline Sleep quality score (PSQI), $\ddagger$ mean (SD) & $4.8(3.0)$ \\
\hline CRP (mg/L), geometric mean (IQR) & $1.1(2.0)$ \\
\hline PAl-1 (ng/mL), mean (SD) & $43(25)$ \\
\hline $\mathrm{TNF} \alpha(\mathrm{pg} / \mathrm{mL})$, mean (SD) & $9.3(2.8)$ \\
\hline IL-6 (pg/mL), geometric mean (IQR) & $1.8(1.6)$ \\
\hline Adiponectin (ug/mL), geometric mean (IQR) & $8.8(8.3)$ \\
\hline HOMA, geometric mean (IQR) & $1.2(0.7)$ \\
\hline QUICKI, mean (SD) & $0.38(0.03)$ \\
\hline MFBF (mL/100 mL tissue/min), mean (SD) & $27(9)$ \\
\hline FBF increase (\%), mean (SD) & $782(311)$ \\
\hline $\begin{array}{l}\text { MFVR (mm Hg/mL/100 } \mathrm{mL} \text { tissue } / \mathrm{min} \text { ), } \\
\text { geometric mean (IQR) }\end{array}$ & $3.0(1.3)$ \\
\hline FVR decrease (\%), geometric mean (IQR) & $86(7)$ \\
\hline HR 24 h (bpm), mean (SD) & $75(10)$ \\
\hline HR awake (bpm), mean (SD) & $77(11)$ \\
\hline HR asleep (bpm), mean (SD) & $64(11)$ \\
\hline HR office (bpm), mean (SD) & $69(11)$ \\
\hline HR 24 h variability (bpm), mean (SD) & $12(4)$ \\
\hline SBP $24 \mathrm{~h}(\mathrm{~mm} \mathrm{Hg})$, mean (SD) & $117(9)$ \\
\hline SBP awake (bpm), mean (SD) & $119(9)$ \\
\hline SBP asleep (bpm), mean (SD) & $105(10)$ \\
\hline SBP office ( $\mathrm{mm} \mathrm{Hg}$ ), mean (SD) & $108(10)$ \\
\hline DBP $24 \mathrm{~h}(\mathrm{~mm} \mathrm{Hg})$, mean (SD) & $72(6)$ \\
\hline DBP awake (bpm), mean (SD) & $74(6)$ \\
\hline DBP asleep (bpm), mean (SD) & $60(6)$ \\
\hline DBP office (mm Hg), mean (SD) & $65(8)$ \\
\hline Prehypertensive (office or 24 h BP), n (\%) & $44(24)$ \\
\hline $\begin{array}{l}\text { White coat prehypertension (office only), } \\
\mathrm{n}(\%)\end{array}$ & $18(10)$ \\
\hline Masked prehypertension (24 h only), n (\%) & $18(10)$ \\
\hline 24 h SBP non-dipper, n (\%) & $66(35)$ \\
\hline
\end{tabular}

${ }^{*}$ Data available for 175 participants.

†Data available for 171 participants.

‡Data available for 117 participants.

$\mathrm{BMI}$, body mass index; CRP, C reactive protein; $\mathrm{DBP}$, diastolic blood pressure; HOMA, homoeostatic model assessment; HR, heart rate; IL-6, interleukin-6; MFBF, maximum forearm blood flow MFVR, minimum forearm vascular resistance; PAI-1, plasminogen activator inhibitor-1; PSQI, Pittsburgh sleep quality index; QUICKI, quantitative insulin sensitivity check index; SBP, systolic blood pressure; TNF, tomour necrosis factor.

unadjusted results were qualitatively similar when office measurements were substituted for mean $24 \mathrm{~h}$ values (not shown), as office and mean $24 \mathrm{~h}$ measures were highly correlated (correlation $=0.67$ for SBP, 0.53 for DBP, 0.74 for HR; all $\mathrm{p}<0.001)$.

Table 3 presents the main results regarding the association of $24 \mathrm{~h}$ HR with $24 \mathrm{~h} \mathrm{SBP}$ and DBP (figure 1, pathway [2]), controlling for age, gender, race,
Table 2 Correlations ( $\mathrm{p}$ values) of $\mathrm{HR}$ and other atherosclerotic risk factors with SBP and DBP $(\mathrm{N}=186)$

\begin{tabular}{lrr}
\hline & \multicolumn{1}{c}{ SBP 24 h } & \multicolumn{1}{c}{ DBP 24 h } \\
\hline HR 24 h & $0.04(0.633)$ & $0.33(0.001)$ \\
HR 24 h variability & $-0.09(0.236)$ & $-0.02(0.830)$ \\
FBF increase & $-0.24(0.001)$ & $-0.09(0.247)$ \\
FVR decrease* & $-0.22(0.002)$ & $-0.08(0.260)$ \\
QUICKI & $-0.16(0.025)$ & $-0.24(0.001)$ \\
CRP $^{*}$ & $0.08(0.289)$ & $0.13(0.075)$ \\
PAI-1 & $0.22(0.003)$ & $0.23(0.002)$ \\
TNF $\alpha$ & $-0.05(0.473)$ & $0.01(0.879)$ \\
IL-6* & $0.05(0.463)$ & $0.07(0.357)$ \\
Adiponectin* & $-0.17(0.018)$ & $-0.05(-0.473)$ \\
BMI & $0.23(0.002)$ & $0.08(0.319)$ \\
WC & $0.34(0.001)$ & $0.19(0.013)$ \\
\hline
\end{tabular}

*Log-transformed.

$\mathrm{BMI}$, body mass index; CRP, C reactive protein; DBP, diastolic blood pressure; FBF, forearm blood flow; FVR, forearm vascular resistance; HR, heart rate; IL-6, interleukin-6; PAI-1, plasminogen activator inhibitor-1; QUICKI, quantitative insulin sensitivity check index; SBP, systolic blood pressure; TNF, tomour necrosis factor; WC, waist circumference.

endothelial function (FBF \% increase from baseline to maximum during postocclusive reactive hyperaemia, figure 1, pathway [2]), and insulin sensitivity (QUICKI, figure 1, pathway [3]). An increment of $10 \mathrm{bpm}$ in the $24 \mathrm{~h} \mathrm{HR}$ (about $1 \mathrm{SD}$ ) was associated with higher $24 \mathrm{~h}$ SBP and DBP, by and average of $1.3 \mathrm{~mm} \mathrm{Hg}(\mathrm{p}=0.042)$ and $1.7 \mathrm{~mm} \mathrm{Hg}(\mathrm{p}=0.001)$, respectively. FBF per cent increase was significantly associated with SBP but not DBP ( $p=0.013$ and 0.499 , respectively), while QUICKI was not associated with either SBP or DBP $(p=0.218$ and 0.283 , respectively).

In additional analyses that adjusted for inflammatory markers and BMI (figure 1, pathways [4] and [5]), the effect of HR on SBP was somewhat attenuated (mean SBP difference $=0.6 \mathrm{~mm} \mathrm{Hg}$ per $10 \mathrm{bpm}$ in $\mathrm{HR}, \mathrm{p}=0.422$ ), but that on DBP remained unchanged (mean DBP difference $=1.7 \mathrm{~mm} \mathrm{Hg}$ per $10 \mathrm{bpm}$ in $\mathrm{HR}, \mathrm{p}=0.001$ ). Furthermore, PAI-1 was also a significant predictor of both SBP (mean difference $=1.4 \mathrm{~mm} \mathrm{Hg}$ per $25 \mathrm{mg} / \mathrm{mL}$ PAI-1, $\mathrm{p}=0.041$ ) and DBP (mean difference $=1.1 \mathrm{~mm} \mathrm{Hg}$ per $25 \mathrm{mg} / \mathrm{mL}$ PAI-1, $\mathrm{p}=0.015)$. Findings were very similar when waist circumference (WC) was substituted for BMI (results not shown).

Awake and asleep HR were both associated with $24 \mathrm{~h}$ SBP ( $\mathrm{p}=0.040$ and 0.068 , respectively) and $24 \mathrm{~h}$ DBP ( $p=0.001$ and 0.062 ). Not surprisingly, the awake HR mean was more strongly associated with the awake SBP and DBP, while the asleep HR mean was more strongly associated with the asleep SBP and DBP. Finally, we did not find any significant association between $24 \mathrm{~h}$ HR variability and $\mathrm{BP}(\mathrm{p}=0.443$ for $\mathrm{SBP}$ and 0.561 for $\mathrm{DBP})$.

The main analyses were also repeated using office measurements of HR and BP instead of their $24 \mathrm{~h}$ ambulatory averages. The associations were generally similar as those seen with ambulatory measurements, although their strength was attenuated by about one-third to 
Table 3 Multivariable results for HR and other atherosclerotic risk factors as determinants of SBP and DBP (N=186)

\begin{tabular}{|c|c|c|c|c|c|c|c|}
\hline & \multirow[t]{2}{*}{ Increment } & \multicolumn{3}{|c|}{ SBP $24 \mathrm{~h}$} & \multicolumn{3}{|c|}{ DBP $24 \mathrm{~h}$} \\
\hline & & $\bar{\Delta}$ & (95\% Cl) & p Value & $\bar{\Delta}$ & (95\% Cl) & p Value \\
\hline HR $24 \mathrm{~h}$ & 10 bpm & 1.3 & (0.0 to 2.6$)$ & 0.042 & 1.7 & (0.8 to 2.5$)$ & 0.001 \\
\hline FBF increase & $300 \%$ & -1.4 & $(-2.5$ to -0.3$)$ & 0.013 & -0.3 & $(-1.0$ to 0.5$)$ & 0.499 \\
\hline QUICKI & 0.03 & -0.8 & $(-2.1$ to 0.5$)$ & 0.218 & -0.5 & $(-1.3$ to 0.4$)$ & 0.283 \\
\hline Age & 10 years & 2.2 & $(0.2$ to 4.1$)$ & 0.033 & 2.8 & (1.5 to 4.1$)$ & 0.001 \\
\hline Gender & $M$ vs $F$ & 9.4 & (6.9 to 11.9 ) & 0.001 & 1.3 & $(-0.4$ to 3.0$)$ & 0.123 \\
\hline Race & Black vs non-black & 0.3 & $(-3.4$ to 4.0$)$ & 0.873 & -0.4 & $(-2.9$ to 2.0$)$ & 0.719 \\
\hline
\end{tabular}

$\triangle$, Mean difference of SBP or DBP (in $\mathrm{mm} \mathrm{Hg}$ ) corresponding to the increment shown for each variable.

DBP, diastolic blood pressure; F, female; FBF, forearm blood flow; HR, heart rate; M, male; QUICKI, quantitative insulin sensitivity check index; SBP, systolic blood pressure.

one-half. As a consequence, neither HR nor PAI-1 was significantly associated with BP, although FBF per cent increase was a significant predictor of office SBP (results not shown).

Table 4 presents the results regarding the association of BMI, inflammatory markers and insulin sensitivity/ resistance with $24 \mathrm{~h}$ HR (figure 1, pathways [6]-[8]). QUICKI showed a very strong inverse association with HR ( $p=0.013)$. Higher CRP was significantly associated with higher HR $(p=0.007)$, while similar associations of PAI-1 and IL-6 with HR were marginally significant ( $p=0.093$ and 0.077 , respectively). Unexpectedly, we also found a significant inverse association between $\mathrm{TNF} \alpha$ and HR ( $\mathrm{p}=0.015)$. Neither BMI nor WC (results not shown) was significantly associated with HR.

\section{DISCUSSION}

Mean $24 \mathrm{~h}$ HR was independently associated with mean $24 \mathrm{~h} \mathrm{SBP}$ and DBP, CRP, and QUICKI. The findings

\begin{tabular}{|c|c|c|c|c|}
\hline & \multirow[b]{2}{*}{ Increment } & \multicolumn{3}{|c|}{ HR 24 h } \\
\hline & & $\Delta$ & $(95 \% \mathrm{Cl})$ & $\begin{array}{l}p \\
\text { Value }\end{array}$ \\
\hline Age & 10 years & -0.6 & $(-2.8$ to 1.7$)$ & 0.617 \\
\hline Gender & $M$ vs $F$ & -6.2 & $(-9.0$ to -3.4$)$ & 0.001 \\
\hline Race & $\begin{array}{l}\text { Black vs } \\
\text { non-black }\end{array}$ & 2.7 & $(-1.7$ to 7.1$)$ & 0.234 \\
\hline BMI & $5 \mathrm{~kg} / \mathrm{m}^{2}$ & 0.4 & ( -1.3 to 2.2$)$ & 0.627 \\
\hline $\mathrm{CRP}^{*}$ & Doubling & 1.3 & (0.4 to 2.3 ) & 0.007 \\
\hline PAI-1 & $25 \mathrm{ng} / \mathrm{Ml}$ & 1.2 & $(-0.2$ to 2.7$)$ & 0.093 \\
\hline TNF $\alpha$ & $3 \mathrm{pg} / \mathrm{mL}$ & -1.7 & $(-3.0$ to -0.3$)$ & 0.015 \\
\hline IL-6* & Doubling & 1.1 & ( -0.1 to 2.4$)$ & 0.077 \\
\hline Adiponectin* & Doubling & 1.0 & ( -0.7 to 2.7$)$ & 0.270 \\
\hline QUICKI & 0.03 & -2.8 & $(-4.4$ to -1.1$)$ & 0.001 \\
\hline
\end{tabular}

$\Delta$, Mean difference of HR (in bpm) corresponding to the increment shown for each variable.

*Log-transformed.

BMI, body mass index; CRP, $C$ reactive protein; $F$, female; HR, heart rate; IL-6, interleukin-6; M, male; PAI-1, plasminogen activator inhibitor-1; QUICKI, quantitative insulin sensitivity check index; TNF, tumour necrosis factor. from this study of young non-hypertensive individuals suggest that $24 \mathrm{~h} \mathrm{HR}$ is associated with known risk factors for atherosclerosis prior to the development of diabetes or sustained hypertension. The magnitude of these associations was modest but still potentially clinically meaningful, particularly given that these young individuals did not exhibit any clinical manifestations of atherosclerosis or cardiovascular disease. Although this study specifically excluded hypertensive individuals, a sizable proportion of participants (35\%) were SBP nondippers, and masked prehypertensives (8\%), possibly indicating the presence of early stage cardiovascular pathology already underway.

In contrast to HR, HR variability was not significantly associated with BP. Previous studies of HR variability demonstrating an association with $\mathrm{BP}^{15}$ and adipokines ${ }^{16}$ were conducted in older individuals, suggesting that HR may be an earlier, more sensitive marker of autonomic dysfunction than HR variability. Alternatively, HR variability is typically determined from $24 \mathrm{~h}$ ECG recordings. However, HR variability has also previously been calculated as the mean of the SD of HR from ABPM reports as in our study. ${ }^{15}$ We chose this methodology as we do not have $24 \mathrm{~h}$ ECG monitoring equipment. ABPM-calculated HR variability was found in the cited reference to be a significant predictor of carotid intimamedia thickness in older hypertensive patients. Our lack of statistically significant findings for HR variability in this study could either be because this alternate methodology may be a less sensitive method for detecting HR variability compared with $24 \mathrm{~h}$ ECG monitoring, or due to the inclusion of younger, healthier patients in this study in whom HR variability may not be pathologically altered. Study findings may have been additionally limited by the moderate sample size of 186 participants.

Many inter-related factors contribute to raised BP. Among the factors that have been intensively studied are salt intake, obesity and insulin resistance, the renin-angiotensin system, the SNS, genetics, endothelial dysfunction, smoking, alcohol intake, and exercise. We have not included all possible determinants of BP in order to limit the complexity of our analyses in this study; we chose to focus on the variables noted in figure 
1: obesity (BMI), insulin resistance, SNS and endothelial dysfunction.

This study presents evidence of an association between elevated SNA, as represented by elevated HR, and atherosclerotic risk factors which have also been implicated in the pathogenesis of elevated BP: endothelial dysfunction, measures of adiposity, insulin resistance and inflammatory cytokines. ${ }^{17}$ Endothelial dysfunction is central to the initiation and progression of atherosclerosis, since vascular endothelial cells secrete several antiatherogenic substances including the potent vasodilator nitric oxide. ${ }^{18}$ Overall, research to date suggests that visceral adiposity is a stronger predictor of endothelial activation than overall adiposity. Insulin resistance is associated with systemic coronary artery disease risk factors, including adiposity; increasing evidence suggests that defective insulin signalling in atherosclerotic lesional cells also contributes to disease progression. ${ }^{19}$

Elevated levels of inflammatory adipokines promote endothelial dysfunction, hypertension and atherosclerosis. ${ }^{17}$ Both CRP and IL-6 are molecular markers associated with atherosclerosis and its progression, with IL-6 showing more consistent results and stronger independent predictive value than other inflammatory markers. ${ }^{20}$ Conclusive in vitro and in vivo findings clearly indicate a proatherogenic role of IL- $6 .{ }^{21}$ IL- 6 signalling has been shown to contribute to both atherosclerotic plaque development and plaque destabilisation, while elevated PAI-1 levels appear to increase the risk of atherothrombotic events and may also promote the progression of vascular disease. ${ }^{22}$

We found that measures of adiposity (BMI or WC) were not significantly associated with HR24 (figure 1, pathway [6]), when insulin resistance and inflammatory markers were controlled for. QUICKI (figure 1, pathway [8]), as well as CRP and to some extent PAI-1 (figure 1, pathway [7]) were independently associated with SNS activity. This suggests that the latter factors may be intermediaries in the main causal pathway linking adiposity to SNS activity. However, we also found an unexpected protective effect of TNF $\alpha$ on HR24 (figure 1, pathway [7]). This could be explained by the fact that plasma $\mathrm{TNF} \alpha$ values are usually low. Measurement of $\mathrm{TNF} \alpha$ receptors, a better marker of TNF $\alpha$ activity, was not part of the study protocol.

Despite the evidence presented in these and other studies, the exact mechanism by which the SNS mediates its deleterious effects on BP remains unknown. Possible additional mechanisms that we did not explore in this study include activation of the renin-angiotensinaldosterone system, impaired baroreceptor reflexes, sleep apnoea-activated chemoreceptor reflexes and adipose tissue-derived leptin-mediated SNS activation. ${ }^{6}$ Also, although our analyses took into account various links between many known or suspected risk factors of atherosclerosis, our data were cross-sectional and cannot establish temporal relationships or causal pathways.
Although multiple epidemiological studies have consistently reported that resting HR is an independent predictor of cardiovascular and all-cause mortality in men and women with and without diagnosed cardiovascular disease, the importance of resting HR as a prognostic factor and potential therapeutic target is not yet generally accepted. Resting HR has been previously found to be a predictor of all-cause mortality in older (aged 45-64 years) prehypertensive older patients. ${ }^{23} \mathrm{HR}$ has also been associated with CRP in elderly men and women (aged 55-75 years), ${ }^{24}$ middle-aged Israeli men (mean age of about 45 years), ${ }^{25}$ and with both insulin resistance and CRP in young Japanese men (aged 18-26 years). ${ }^{26}$ For this study, we specifically recruited young non-hypertensive individuals (including normotensives and prehypertensives) as we aimed to study individuals who have not yet progressed to clinical disease. An additional strength of our study is its diverse population, with substantial fractions of both African-Americans and Asians. African-Americans have not been well studied regarding ambulatory monitoring, although hypertension occurs more commonly and is associated with greater morbidity and mortality in African-Americans compared with Caucasians. ${ }^{27}$

Most previous studies of HR have utilised resting HR. HR recorded over $24 \mathrm{~h}$ has previously been reported to have a better reproducibility (correlation 0.73, with a mean change of only $0.7 \mathrm{bpm}$ over a 3 -month interval in 839 hypertensive patients) than office HR (Palatini et al, 2000). Twenty-four-hour HR could therefore be a better prognostic indicator than traditional measurement of resting HR.

Three previous studies analysed mean $24 \mathrm{~h} \mathrm{HR}$ in individuals much older than those in our study, one with patients with a mean age of $52,{ }^{28}$ and the other among patients aged 55-75 years. ${ }^{29}$ The large ABP-International study of 7600 Caucasians and Asians reported independent predictive value for night-time HR, but not daytime or $24 \mathrm{~h} \mathrm{HR}$, for cardiovascular, but not all-cause mortality. ${ }^{28}$ Similar findings were reported in a study of 653 elderly Europeans. ${ }^{29}$ However, ambulatory HR did not add additional prognostic information over office HR in a third study of nearly 5000 elderly hypertensive Europeans. ${ }^{30}$ In our analyses, associations were markedly stronger for $24 \mathrm{~h}$ measurements than for office (resting) measurements, suggesting that the $24 \mathrm{~h}$ measures may be more sensitive than their office (resting) counterparts in young non-hypertensive adults.

\section{CONCLUSIONS}

In conclusion, HR, an established indicator of SNS activity, was associated with elevated BP, CRP and insulin resistance in non-hypertensive individuals. This finding suggests that HR may be a potential marker of elevated cardiovascular risk in asymptomatic individuals, prior to the development of clinical hypertension or cardiovascular disease. Furthermore, our findings suggest that the link of adiposity with HR may be mediated by 
inflammatory markers and insulin resistance. Prospective longitudinal studies are needed to further elucidate the causal pathways that link adiposity, inflammation, insulin resistance, endothelial function and HR, and the degree to which HR measured in early life can predict the incidence of atherosclerosis and cardiovascular disease. Clinical strategies incorporating HR in risk stratification may have possible future utility in identifying asymptomatic individuals to target with individualised preventive therapy.

Contributors Both CC and CD contributed to the work by meeting all four of the following authorship criteria: substantial contributions to the conception or design of the work; also, the acquisition, analysis or interpretation of data for the work; drafting the work or revising it critically for important intellectual content; final approval of the version to be published; agreement to be accountable for all aspects of the work in ensuring that questions related to the accuracy or integrity of any part of the work are appropriately investigated and resolved.

Funding Funding for this study was provided by NIH grant HL096593.

Competing interests None declared.

Ethics approval Thomas Jefferson University Institutional Review Board.

Provenance and peer review Not commissioned; externally peer reviewed.

Data sharing statement Extra data is available by emailing CC at Cynthia.Cheng@jefferson.edu.

Open Access This is an Open Access article distributed in accordance with the Creative Commons Attribution Non Commercial (CC BY-NC 4.0) license, which permits others to distribute, remix, adapt, build upon this work noncommercially, and license their derivative works on different terms, provided the original work is properly cited and the use is non-commercial. See: http:// creativecommons.org/licenses/by-nc/4.0/

\section{REFERENCES}

1. Custodis F, Schirmer SH, Baumhakel M, et al. Vascular pathophysiology in response to increased heart rate. J Am Coll Cardiol 2010;56:1973-83.

2. Oparil S, Zaman MA, Calhoun DA. Pathogenesis of hypertension. Ann Intern Med 2003;139:761-76.

3. Mancia G, Grassi G, Giannattasio C, et al. Sympathetic activation in the pathogenesis of hypertension and progression of organ damage. Hypertension 1999;34(4 Pt 2):724-8.

4. Julius $\mathrm{S}$, Nesbitt $\mathrm{S}$. Sympathetic overactivity in hypertension. A moving target. Am J Hypertens 1996;9:113S-20S.

5. Grassi G. Sympathetic neural activity in hypertension and related diseases. Am J Hypertens 2010;23:1052-60.

6. Hall JE, da Silva AA, do Carmo JM, et al. Obesity-induced hypertension: role of sympathetic nervous system, leptin, and melanocortins. J Biol Chem 2010;285:17271-6.

7. Esler M, Straznicky N, Eikelis N, et al. Mechanisms of sympathetic activation in obesity-related hypertension. Hypertension 2006;48:787-96.

8. Julius $S$, Valentini M, Palatini P. Overweight and hypertension: a 2-way street? Hypertension 2000;35:807-13.
9. Malpas SC. Sympathetic nervous system overactivity and its role in the development of cardiovascular disease. Physiol Rev 2010;90:513-57.

10. Alvarez GE, Beske SD, Ballard TP, et al. Sympathetic neural activation in visceral obesity. Circulation 2002;106:2533-6.

11. Thorin E, Thorin-Trescases N. Vascular endothelial ageing heartbeat after heartbeat. Cardiovasc Res 2009;84:24-32.

12. Schulte KL, Braun J, Meyer-Sabellek W, et al. Functional versus structural changes of forearm vascular resistance in hypertension. Hypertension 1988;11:320-5.

13. Sivertsson R. The hemodynamic importance of structural vascular changes in essential hypertension. Acta Physiol Scand Suppl 1970;343:1-56.

14. Cheng $\mathrm{C}$, Campbell $\mathrm{KL}$, Kushner $\mathrm{H}$, et al. Correlation of oral glucose tolerance test-derived estimates of insulin sensitivity with insulin clamp measurements in an African-American cohort. Metab Clin Exp 2004;53:1107-12.

15. Garcia-Garcia A, Gomez-Marcos MA, Recio-Rodriguez JI, et al. Office and 24-hour heart rate and target organ damage in hypertensive patients. BMC Cardiovasc Disord 2012;12:19.

16. Lampert R, Bremner JD, Su S, et al. Decreased heart rate variability is associated with higher levels of inflammation in middle-aged men. Am Heart J 2008;156:759.e1-7.

17. Mattu HS, Randeva HS. Role of adipokines in cardiovascular disease. J Endocrinol 2013;216:T17-36.

18. Kerr SM, Livingstone MB, McCrorie TA, et al. Endothelial dysfunction associated with obesity and the effect of weight loss interventions. Proc Nutr Soc 2011;70:418-25.

19. Bornfeldt KE, Tabas I. Insulin resistance, hyperglycemia, and atherosclerosis. Cell Metab 2011;14:575-85.

20. Tzoulaki I, Murray GD, Lee AJ, et al. C-reactive protein, interleukin-6, and soluble adhesion molecules as predictors of progressive peripheral atherosclerosis in the general population: Edinburgh Artery Study. Circulation 2005;112:976-83.

21. Schuett $H$, Luchtefeld $M$, Grothusen $C$, et al. How much is too much? Interleukin-6 and its signalling in atherosclerosis. Thromb Haemost 2009;102:215-22.

22. Vaughan DE. PAl-1 and atherothrombosis. J Thromb Haemost 2005;3:1879-83.

23. King DE, Everett CJ, Mainous AG III, et al. Long-term prognostic value of resting heart rate in subjects with prehypertension. $A m \mathrm{~J}$ Hypertens 2006;19:796-800.

24. Sajadieh A, Nielsen OW, Rasmussen V, et al. Increased heart rate and reduced heart-rate variability are associated with subclinical inflammation in middle-aged and elderly subjects with no apparent heart disease. Eur Heart $J$ 2004;25:363-70.

25. Rogowski O, Shapira I, Shirom A, et al. Heart rate and microinflammation in men: a relevant atherothrombotic link. Heart 2007;93:940-4.

26. Kazumi T, Kawaguchi A, Sakai K, et al. Young men with high-norma blood pressure have lower serum adiponectin, smaller LDL size, and higher elevated heart rate than those with optimal blood pressure. Diabetes Care 2002;25:971-6.

27. Mensah GA, Brown DW. An overview of cardiovascular disease burden in the United States. Health Aff (Millwood) 2007;26: 38-48.

28. Palatini P, Reboldi G, Beilin LJ, et al. Predictive value of night-time heart rate for cardiovascular events in hypertension. The ABP-International study. Int J Cardiol 2013;168:1490-5.

29. Johansen $C D$, Olsen $\mathrm{RH}$, Pedersen LR, et al. Resting, night-time, and $24 \mathrm{~h}$ heart rate as markers of cardiovascular risk in middle-aged and elderly men and women with no apparent heart disease. Eur Heart J 2013;34:1732-9.

30. Palatini $P$, Thijs $L$, Staessen JA, et al. Predictive value of clinic and ambulatory heart rate for mortality in elderly subjects with systolic hypertension. Arch Intern Med 2002;162:2313-21. 